\title{
Validation of pore network model for hygric property calculation
}

\author{
Muhammad Islahuddin, Chi Feng, Steven Claes and Hans Janssen \\ KU Leuven, Department of Civil Engineering, Building Physics Section, Kasteelpark Arenberg 40, \\ 3001 Leuven, Belgium
}

\begin{abstract}
Hygric properties can be estimated directly from pore structure information, represented by a network of regularly shaped pores, extracted from a pore structure image to conserve the real topology. On this network, pore-scale models of moisture behaviour determine the hygric properties of moisture storage and transport. The reliability of this approach is validated with a sintered-glass filter. Despite its more limited heterogeneity and pore size range relative to typical porous building materials, it provides a good basis for validating crucial pore-scale moisture processes. Measured storage data compare well to the estimated ad- and desorption moisture retention curves as well as to the saturated and capillary moisture content. Furthermore, the simulated whole-range moisture permeability curve agrees acceptably with measured data. The variation in modelling the pore space as a pore network model is also analysed by considering two distinct pore network extraction methods. The measured and simulated moisture contents agree well for the whole capillary range. Moreover, the resulting transport properties are generally accurate for the whole moisture content range. On the other hand, the estimated vapour permeabilities show notable variations between the two pore network models.
\end{abstract}

\section{Introduction}

A reliable moisture assessment of building components is crucial in designing sustainable and durable built structures and in creating healthy and comfortable living environments. Such an assessment requires the hygric properties of the building materials, whose accuracy determines the assessment reliability. An accurate determination of the hygric properties is hence crucial.

As an alternative to measurements, numerical studies have been proposed to determine the hygric properties of building materials from a network of pores and throats constructed from their basic pore structure information. [1] constructed a regular two-dimensional pore network model with a presumed pore size distribution to determine sorption isotherms and water vapour permeabilities. [2] improved the method by incorporating measured moisture storage functions as well as permeabilities at low and capillary saturation levels to estimate the unsaturated permeability curve.

The current state-of-the-art approach constructs a representative pore network model from a pore space image conserving its topological and geometrical information [3-4]. Pore-scale physical storage and transport models are then applied to the resulting real-sized 
pore network to simulate the moisture-related processes. However, this approach currently is still limited to an exemplary study and has not been validated yet.

This study is therefore conducted to validate such a topological pore network approach for the estimation of moisture storage and transport properties of building materials. The discussion begins with a brief summary of the topological pore network model proposed in [5]. The following section discusses its validation using a sintered-glass filter. Despite its smaller heterogeneity and pore size range relative to typical porous building materials, it provides a good basis for testing several crucial moisture-related processes. The confrontation of measured and estimated moisture storage and moisture transport properties then concludes the paper.

\section{Pore network model}

The moisture storage and moisture transport properties are numerically determined by the quasi-static pore network model, a more detailed presentation of which can be found in [5]. For completeness of this paper, the model is briefly summarised here.

The moisture storage is determined by the aggregate volume of condensed water and of corner and surface films present in the network. This results in an accurate estimation, since the volumetric information has been conserved from the pore space image. The calculation of the moisture content is performed after determining the condensed water distribution by the Kelvin equation. In dry pores, the amount of corner water is derived geometrically [6], while the amount of surface water is determined statistically with the Bradley equation [7]. These calculations are done for a series of imposed capillary pressures, finally resulting in a moisture retention curve. Depending on the applied initial moisture content and invasion or drainage algorithm, adsorption, desorption and even scanning curves can be obtained.

From the moisture distribution obtained for a particular capillary pressure, the moisture transport is determined by invoking a stationary moisture flow via an infinitesimal pressure difference between two opposing boundaries. This moisture flows in liquid form in waterfilled elements as well as via the corner and surface films in the dry elements. Additionally, water vapour diffusion takes place in the dry elements. The stationary flow implies that the moisture masses flowing in and out of each pore are identical. This yields a system of linear equations with the capillary pressures at the different pores as unknowns and the overall liquid and vapour conductance between two pores as the constants. Solving these equations allows to calculate the global flow through the in- and outlet plane to finally determine the permeability using the Darcy equation.

\section{Validation}

To validate the hygric property model, numerical simulations are compared to experimental results for a ROBU P100 sintered-glass filter. This material has been preferred over an actual building material, given that it only has coarse pores ranging from 2 to 130 micrometers, hence allowing a reliable characterization of its pore structure via X-ray CT [8]. To transform the obtained pore structure to a pore network model, the original maximal-ball (MB) network extraction [9] and its recently revised version [10] are used, illustrating the variability due to the network extraction. The new extraction has been adjusted in the pore-throat definition and in the calibration to single-phase flow simulations in sandstone rocks. Relative to the old code, the new code yields fewer pore elements as shown by the pore size distributions in Figure 1. Since the related throat volumes are significantly higher, it appears that the new code tends to lump small elements together into throats. The new code moreover produces more regular triangular pores while the old code 
gives more slit-shaped triangles. The measurements performed on the glass filter include mercury intrusion porosimetry (MIP) to determine the desorption moisture retention curve, free water uptake testing to obtain the capillary moisture content and the moisture diffusivity, hanging-water-column experiments to find the near-saturation moisture permeabilities and cup vapour transmission measurements to determine the vapour permeabilities. These measurements unfortunately do not cover the full range of applicability of the hygric property model, but the current limitations on experimental hygric property determination do not allow a more complete validation.

(a)

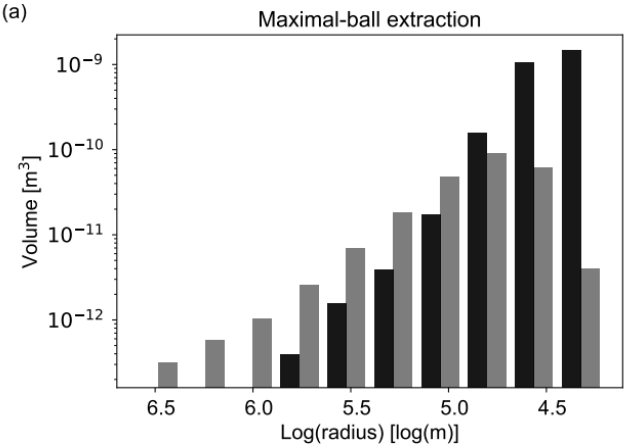

(b)

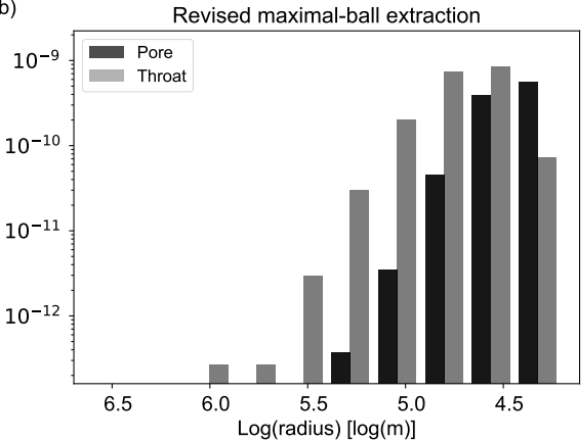

Fig. 1. Pore volume distribution of the glass filter networks resulted by the original (left) and revised (right) maximal-ball network extraction codes, respectively.

\subsection{Moisture storage}

Figure 2(a) depicts the measured and simulated desorption moisture retention curves for the glass filter. The variability in the experimental data may originate from differences in sample sizes, in measuring procedures and/or equilibrium criteria [11, 12]. As mercury does not spontaneously adsorb onto pore walls or condense into pore corners, the simulation excludes corner and surface films. In general, the simulations and measurements agree well, certainly for the new extraction code. The differences between the two distinct codes reflect the differences in the pore radii distributions, see Figure 1. The small difference of the saturated moisture content originates from the deviating handling of boundary pores: the bigger pore volume of the new code results in a higher volume adjustment. Figure 2(b) compares the measured and simulated capillary moisture content, plus the entire adsorption moisture retention curve from the simulation. In this simulation, an imbibition process with air entrapment is calculated. For both extraction codes, the simulated capillary moisture contents are at the high end of the measured results. One should keep in mind though that the capillary absorption in this rough-porous material is probably hindered by gravity [13], an effect not considered in the simulations. From the shown measured results, the highest two values stem from short samples $(3 \mathrm{~cm}$ high), which are least affected by gravity. In relation to these values, the overestimation by the simulations is only $3 \%$ or $7 \%$ for the old and new code respectively, which is far below the differences typically found when doing inter-laboratory evaluations [12].

\subsection{Moisture transport}

Figures 2(c-d) compare the measured and simulated moisture permeabilities: Figure 2(c) presents moisture permeabilities in the high-saturation range, Figure 2(d) shows vapour 
permeabilities in the low-saturation range. The old extraction code typically gives overestimations with a factor 2 to 2.5 . The new code on the other hand yields a very good agreement between experiments and simulations. This new code has been calibrated externally for single-phase flow simulations in sandstone rocks [10], and the flow regimes in these high- and low-saturation ranges resemble single-phase flow in building materials quite much. Figures 2(e-f) finally compare measured and simulated diffusivities. The measured diffusivities are derived from X-ray-visualised moisture content profiles during a free water uptake test [14]. The simulated diffusivities are obtained from the moisture retention and permeability curves, calculated with the imbibition invasion algorithm. These diffusivities are determined based on the Eq. 1:

$$
D=k /\left(\mathrm{d} w / \mathrm{d} p_{\mathrm{c}}\right)
$$

with $D\left[\mathrm{~m}^{2} / \mathrm{s}\right]$ the diffusivity, $k[\mathrm{~kg} /(\mathrm{msPa})]$ the moisture permeability, and $\mathrm{d} w / \mathrm{d} p_{c}$ $\left[\mathrm{kg} /\left(\mathrm{m}^{3} \mathrm{~Pa}\right)\right]$ the moisture capacity, being the derivative of the moisture retention curve. 
(a)

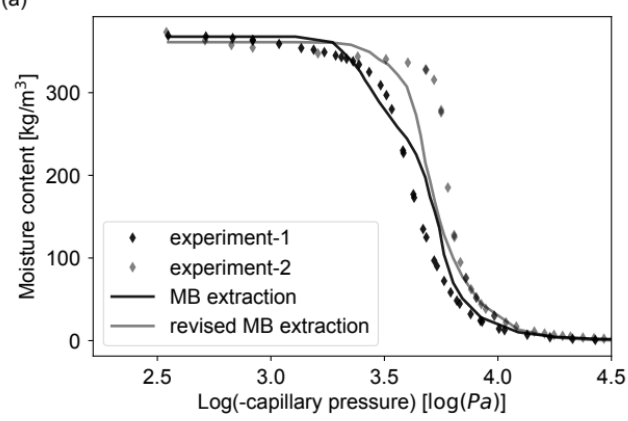

(c)

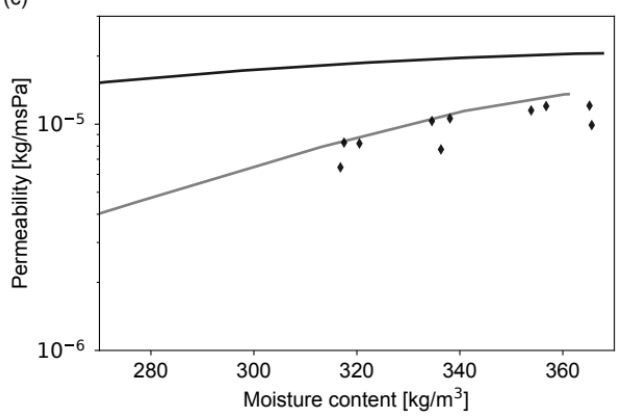

(e)

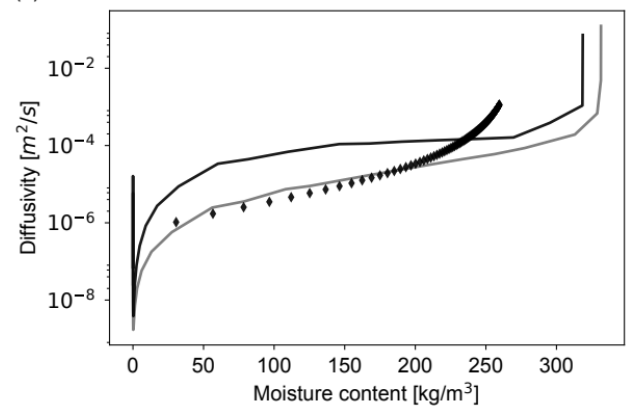

(b)

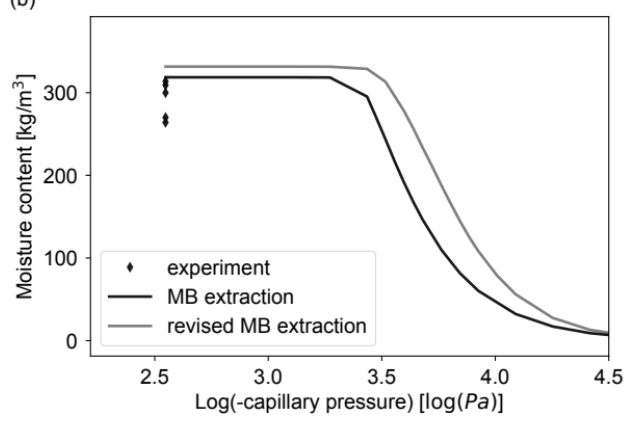

(d)

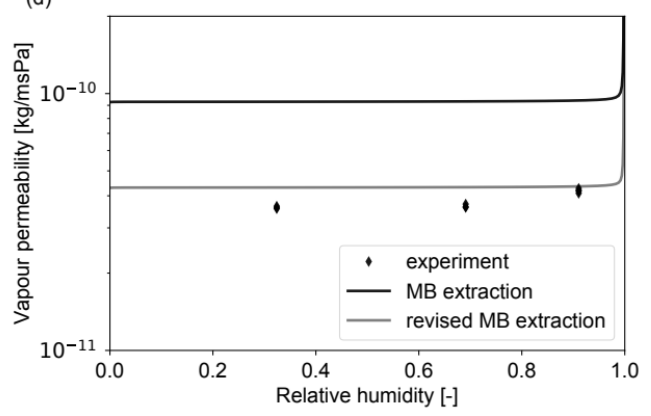

(f)

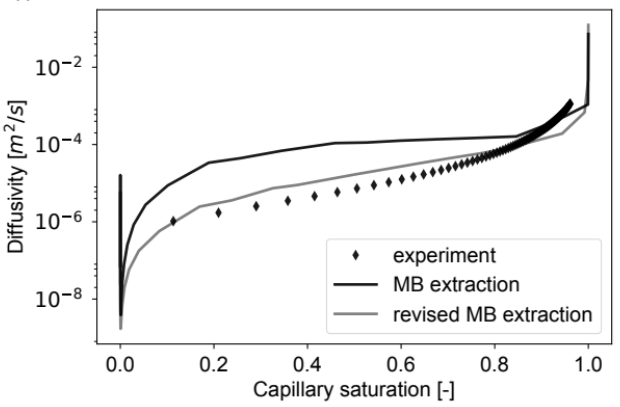

Fig. 2. Measured (dot) and simulated (line curves) properties of the sintered glass: (a) MIP data and moisture retention curves of desorption, (b) Capillary absorption data and moisture retention curves of imbibition with air entrapment, (c) Permeability - moisture content data and curves of adsorption in the over capillary - saturated region, (d) Vapour permeability - relative humidity data and curves of adsorptions, (e-f) Diffusivity data and curves of imbibition with air entrapment with respect to: (e) the moisture content, (f) the capillary saturation.

Figure 2(e) depicts the diffusivities in function of moisture content, Figure 2(f) uses the ratio of the moisture content over the capillary moisture content. The latter presentation aims at correcting for the difference in the measured and simulated capillary moisture contents: $270 \mathrm{~kg} / \mathrm{m}^{3}$ in the measurement, due to the use of a high sample, versus $318 \mathrm{~kg} / \mathrm{m}^{3}$ and $332 \mathrm{~kg} / \mathrm{m}^{3}$ in the simulation. As before, there is a good agreement for the new extraction, while the old extraction leads to an overestimation. At very low moisture contents the simulated diffusivities show the typical 'dip' [15], which experimentally cannot be obtained by free water uptake tests however; instead drying experiments are required. In the proposed hygric property model, this typical profile is a direct result from the simulation of unsaturated moisture storage and transport at all moisture contents. At the higher moisture contents, the deviations between the measured and simulated diffusivities 
are somewhat higher, which is probably highly related to the deviations between the measured and simulated capillary moisture content, see above.

\section{Conclusions}

The simulation results of moisture storage are in good match with the measured values for the whole capillary range. This also includes the capillary moisture content which validates the imbibition and air entrapment algorithms. Moreover, the simulated transport properties in general resemble the experimental data. At near-saturated conditions, they are a good match, consistent with other reported validations. A notable issue in hygroscopic condition is however found. The estimation values of vapour permeability consistently overestimate the data over the whole hygroscopic range by a constant ratio.

The satisfaction results of moisture content suggest that the pore space is accurately quantified and the moisture invasion procedure is valid. The topology of the pore network is also confirmed by the accurate capillary moisture content which is highly dependent on the topological information. However, the geometrical simplification and its concept of shape factor are heavily questioned for producing less accurate vapour permeability.

Given the fine agreement between the measured and simulated (based on the new code) moisture storage and transport properties, the validation of the hygric property model for the glass filter can be considered successful. Such successful validation supports the potential of pore-structure-based simulation of the unsaturated hygric properties of porous materials. It should be noted though that the glass filter is a fairly coarse-porous material, and that the model still is to be validated for actual building materials, with typically also fine-porous porosity present. The conditions at low capillary pressures hence remain to be verified.

This project is supported by the FWO Odysseus grant "Moisture transfer in building materials: analysis at the pore-scale level".

\section{References}

1. D. Quenard, \& H. Sallee, Materials and Structures 25, 515-522 (1992)

2. J. Carmeliet, F. Descamps, G. Houvenaghel, Transp. Porous Media 35, 67-88 (1999)

3. M. Islahuddin, H. Janssen, Proc. CESBP Cent. Eur. Symp. Build. Phys. BauSIM, 301308 (2016)

4. M. Islahuddin, H. Janssen, Energy Procedia 132, 273-278 (2017)

5. M. Islahuddin, H. Janssen, Transp. Porous Media, (to be published)

6. G. Mason, N.R. Morrow, J. Colloid Interface Sci. 141, 262-274 (1991)

7. R. Badmann, N. Stockhausen, M.J. Setzer, J. Colloid Interface Sci. 82, 534-542 (1981)

8. W. Van De Walle, S. Claes, H. Janssen, Constr. Build. Mater. 182, 427-440 (2018)

9. H. Dong, M.J. Blunt, Phys. Rev. E. 80, 036307 (2009)

10. https://github.com/aliraeini/pnextract, accessed May 15, 2018

11. S. Roels, J. Carmeliet, H. Hens, O. Adan, H. Brocken, R. Cerny, Z. Pavlik, C. Hall, K. Kumaran, L. Pel, R. Plagge, J. Build. Phys. 27, 307-325 (2004)

12. S. Roels, J. Carmeliet, H. Hens, HAMSTAD WP1, (2003)

13. N. Fries, M. Dreyer, J. Colloid Interface Sci. 320, 259-263 (2008) 
14. J. Carmeliet, H. Hens, S. Roels, O. Adan, H. Brocken, R. Cerny, Z. Pavlik, C. Hall, K. Kumaran, L. Pel, J. Build. Phys. 27, 277-305 (2004)

15. L. Pel, K. Kopinga, H. Brocken, Heron 41, 95-105 (1996) 